J. Clin. Chem. Clin. Biochem.

Vol. 18, 1980, pp. 137-143

\title{
Bindungskapazität von sexualhormon- und corticosteroidbindendem Globulin im Serum bei Männern mit Lebercirrhose ${ }^{1}$ )
}

\author{
Von $H$. Kaulhausen \\ Frauenklinik der Universität Bonn,
}

H. G. Rohner

Medizinische Klinik der Universität Bonn,

\section{Siedek}

Chirurgische Klinik der Universität Bonn,

M. Lafosse und H. Breuer,

Institut für Klinische Biochemie der Universität Bonn

(Eingegangen am 17. Mai/14. September 1979)

Zusammenfassung: Bei 63 Männern mit Lebercirrhose und bei 42 männlichen Normalpersonen wurden die Bindungskapazitäten von sexualhormonbindendem Globulin (SHBG) und corticosteroidbindendem Globulin (CBG) mit Hilfe der Agargelelektrophorese bestimmt. Der Normalbereich $(\bar{x} \pm 2 \mathrm{~s})$ lag für SHBG zwischen 8,3-17,1 $\mu \mathrm{g} / \mathrm{l}$, für CBG zwischen 46,4-82,8 $\mu \mathrm{g} / \mathrm{l}$. Bei den Patienten mit Lebercirrhose war die Bindungskapazität von SHBG signifikant erhöht $(\overline{\mathrm{x}}=18,1 \mu \mathrm{g} / \mathrm{l})$, während die Bindungskapazität von CBG erniedrigt war $(\overline{\mathrm{x}}=49,7 \mu \mathrm{g} / 1)$. Eine Korrelation zwischen beiden Transportproteinen wurde nicht beobachtet; allerdings war bei Patienten mit niedrigem CBG auch SHBG relativ niedrig. Zwischen der Gesamteiweißkonzentration im Serum, der Aktivität der Cholinesterase und den Gerinnungsfaktoren V und VII als weiteren Kenngrößen der Proteinsynthese einerseits und den Bindungskapazitäten von SHBG und CBG andererseits bestand ebenfalls keine Korrelation. Im Gegensatz zur Lebercirrhose konnte bei Patienten mit einer Fettleber $(n=12)$ oder einer Fibrose der Leber $(n=11)$ kein Unterschied in den Bindungskapazitäten von SHBG und CBG gegenüber Normalpersonen festgestellt werden. Eine Behandlung mit täglich $200 \mathrm{mg}$ Spironolacton über sieben Tage führte bei neun Versuchṣperșonen zu kẹnen Veränḍerungen der Bindungskapazitäten von SHBG und CBG.

\section{Binding capacity of sex hormone binding globulin and corticosteroid binding globulin in serum of male patients with liver cirrhosis}

Summary: The binding capacities of SHBG and CBG were measured by agar gel electrophoresis in 63 men with cirrhosis of the liver and in 42 healthy male subjects. The normal range $(\bar{x} \pm 2 s)$ for SHBG was $8.3-17.1 \mu \mathrm{g} / \mathrm{l}$, for CBG 46.4-82.8 $\mu \mathrm{g} / 1$. SHBG binding capacity was significantly higher in men with liver cirrhosis (mean $18.1 \mu \mathrm{g} / \mathrm{l}$; $\mathrm{p}<0.001$ ) but CBG binding capacity was significantly lower (mean $49.7 \mu \mathrm{g} / 1 ; \mathrm{p}<0.001$ ). Although SHBG was lower in patients with decreased CBG binding capacity, a correlation between both steroid binding proteins did not exist. Moreover, there was no correlation between SHBG or CBG on one hand and other parameters of hepatic protein synthesis such as serum protein concentration, cholinesterase activity and the coagulation factors V and VII on the other hand. In contrast to liver cirrhosis, 12 patients with fatty liver and 11 patients with toxic fibrosis of the liver did not reveal changes in SHBG or CBG. Treatment with spironolactone (200 mg daily for one week in 9 subjects) did not change the steroid binding capacity of human serum.

\section{Einfüitirung}

Gynäkomastie, Hodenătrophie, Libido- und Potenzstörungen, Behaarungsanomalien, Spinnennaevi und Palmạerythem bei Männern mit Lebercirrḥoșe werden als Folge endokriner Veränderungen im östrogen- und

\footnotetext{
1) Auszugsweise vorgetragen von $H$. Breuer auf der gemeinsamen Tagung der Deutschen, Österreichischen und Schweizerischen Gesellschaften für Klinische Chemie, Freiburg, 1975(1).
}

Androgen-Stoffwechsel betrachtet, seitdem Edmondson und Mitarbeiter $(2,3)$ erstmals bei diesem Krankheitsbild eine erhöhte Ausscheidung von Östrogenen im Urin nachgewiesen hatten. Ihre Befunde wurden zwar von zahlreichen anderen Autoren bestätigt, eine Korrelation zwischen dem Anstieg der Östrogenausscheidung und dem Auftreten endokriner Symptome, wie z.B. einer Gynäkomastie, konnte jedoch nicht gefunden werden (4-7). Auch die später von mehreren Untersuchern 
nachgewiesene erhöhte Konzentration von Östradiol$17 \beta$ im Plasma von Männern mit Lebercirrhose (8-12) war allein keine ausreichende Erklärung für das Auftreten der Symptome eines Hypogonadismus, da der Anteil von nicht proteingebundenem und somit biologisch aktivem Östradiol-17 $\beta$ im Plasma bei Patienten mit Lebercirrhose und anderen chronischen Leberkrankheiten gegenüber männlichen Normalpersonen unverändert war (13).

Im Zusammenhang mit der Beschreibung einer niedrigen Konzentration von Testosteron $(8-10,14-16)$ und $5 \alpha$-Dihydrotestosteron (9) im Plasma von Männern mit Lebercirrhose war der Nachweis einer gleichzeitig erhöhten Bindungskapazität des sexualhormonbindenden Globulins (SHBG) im Plasma durch Tavernetți et al. (17) von besonderer Bedeutung; denn dieses $\beta$-Globulin bindet reversibel und mit hoher Affinität, also spezifisch, sowohl $5 \alpha$-Dihydrotestosteron als auch Testosteron, während Östradiol-17 $\beta$ deutlich weniger (etwa $50 \%$ ) gebunden wird. Da die Synthese von SHBG in der Leber darüber hinaus durch Östrogene gesteigert wird, führt ein Anstieg der SHBG-Konzentration im Plasma vor allem zu einer Verminderung des Anteils an biologisch aktiven Androgenen im Plasma; SHBG wird deshalb als indirekter Verstärker der Östrogenwirkung angesehen (18).

In der Zwischenzeit wurde auch von anderen Arbeitsgruppen $(13,19-21)$ eine erhöhte Bindungskapazität von SHBG bei Lebercirrhose beschrieben. Die untersuchten Kollektive waren jedoch zu klein, um mögliche Korrelationen zwischen den Veränderungen der SHBG-Konzentration im Plasma und der Ausprägung der klinischen Symptome eines Androgenmangels - sofern überhaupt beschrieben - überprüfen zu können. Die im folgenden dargelegten Unterșuchungen an 63 Patienten zweier Universitätskliniken sollen zur Klärung folgender Fragen beitragen:

1. Lassen sich Patienten mit deutlich bzw. schwach ausgeprägten endokrinen Symptomen aufgrund einer erhöhten bzw. normalen SHBG-Bindungskapazität unterscheiden?

2. Sind bestimmte Symptome, wie z.B. die Gynäkomastie, enger mit den SHBG-Veränderungen zu korrelieren als andere, z.B. Spinnennaevi und Palmarerythem?

3. Ist die SHBG-Konzentration im Plasma bei der alkoholbedingten Lebercirrhose höher als bei der posthepatitischen Cirrhose?

Da die Synthese des corticosteroidbindenden Globulins (CBG) ebenfalls durch Östrogene stimuliert wird (22), erschien es wichtig, auch die CBG-Konzentration im Plasma bei Lebercirrhose zu untersuchen; Sandberg \& Slaunwhite (22) hatten bei 15 männlichen Patienten keine signifikanten Veränderungen festgestellt. In der Zwischenzeit wurden bei weiteren 15 Männern mit Lebercirrhose sowohl normale Konzentrationen von Cortisol als auch eine unveränderte prozentuale Bindung dieses Steroids im Plasma gefunden (10).

\section{Methodik}

Versuchspersonen

Die SHBG- und CBG-Bindungskapazitäten wurden bei 43 bzw. 42 männlichen Normalpersonen sowie bei 63 männlichen Patienten mit Lebercirrhose bestimmt. Bei den lebergesunden Probanden handelte es sich um 14 bis dahin unbehandelte Patienten einer neurologisch-psychiatrischen Poliklinik, bei den übrigen um Krankenpfleger, Studenten und Ärzte. Bei allen Normalpersonen hatten sich anamnestisch und klinisch keine Anhaltspunkte für Krankheiten der Leber und Schilddrüse oder chronische Leiden ergeben.

Die Patienten wurden entweder in der Medizinischen Klinik $(n=32)$ oder in der Chirurgischen Klinik $(n=31)$ der Universität Bornn betreut. Entsprechend dẹr allgemeinen Zusammensetzung der in diesen Kliniken behandelten Patienten handelte es sich um fortgeschrittene oder im Verlauf ungünstige Fälle von Lebercirrhose (genauere Aufschlüsselung vgl. Ergebnisse). Die Diagnose einer Lebercirrhose war bei allen Patienten histologisch und/oder laparoskopisch gesichert worden.

Klinisch-endokrine Symptome bei Lebercirrhose

Die Patienten der Medizinischen und Chirurgischen Universitätsklinik wurden von jeweils demselben Untersucher (H.G.R. bzw. M.S.) über die Art der Studie aufgeklärt und nach Erhebung der Anamnese bezüglich der endokrinen Symptome befragt und untersucht.

Als Gynäkomastie wurde ein beidseitig (in Einzelfällen auch einseitig) als scheibenförmige subareoläre Induration tạstbares Brustdrüsengewebe bezeichnet; eine bei mehreren Patienten erkennbare, nur auf vermehrter Fetteinlagerung beruhende Brustvergrößerung, die sog. Pseudogynäkomastie wurde nicht berücksichtigt. Für andere Ursachen einer Gynäkomastie ergaben sich keine Anhaltspunkte mit Ausnahme der gesondert angegebenen, vorausgegangènen Einnahme von Spirónolacton. In einigen Fällen fiel die Gynäkomastie den Patienten erst nach Beginn der Therapie mit Spironolacton auf. Bei den Patienten mit geringgradiger oder fraglicher Gynäkomastie wurde eine Mammographie durchgefuhrt, die jeweils mit dem klinischen Eindruck übereinstimmende Ergebnisse ergab.

Die Hodengröße wurde mittels einer Schieblehre gemessen. Als untere Grenzwerte der normalen Hodengröße wurden $4 \times 3$ $X 2,5 \mathrm{~cm}$ Durchmesser $(\mathrm{a} \cdot \mathrm{b} \cdot \mathrm{c})$ angesehen, was einem Volumen von $15,7 \mathrm{ml}$ entspricht $(\mathrm{V}=1 / 6 \cdot \mathrm{a} \cdot \mathrm{b} \cdot \mathrm{c})$ und somit mit der von Prader (23) angegebenen unteren Grenze für ein normales Hodenvolumen des Erwachsenen von $15,0 \mathrm{ml}$ gut übereinstimmt.

In einem Gespräch wurde versucht, verwertbare Angaben über Veränderungen von Libido und Potenz vor Erkennung und im Verlauf der Erkrankung zu erhalten. Auch nach einer etwaigen Verminderung der Sekundärbehaarung (Stamm-, Achsel- und Schambehaarung) wurde gefragt, wobei jeweils darauf geachtet wurde, daß eine spärliche Behaarung nicht schon präexistent gewesen war.

Darüber hinaus wurden die Patienten auf das Vorliegen von Gefäßspinnen (Spider naevi) und eines Palmarerythems untersucht.

\section{Bestimmungsmethoden}

Die Bindungskapazität von SHBG und CBG im Serum wurde mittels der Agargelelektrophorese nach Wagner (24) bestimmt. Dabei wurden zunächst die in der jeweiligen Serumprobe enthaltenen Steroide durch Adsorption an Aktivkohle $(25 \mathrm{mg} / \mathrm{ml}$ 1:2 mit $9 \mathrm{~g} / 1 \mathrm{NaCl}$-Lösung verd. Serum) entfernt; die Probe wurde nach Zentrifugation, Dekantierung und Filtration entweder sofort weiterverarbeitet oder bei $-25^{\circ} \mathrm{C}$ eingefroren. $1 \mathrm{ml}$ einer 1:50 mit $9 \mathrm{~g} / 1 \mathrm{NaCl}-\mathrm{L}$ ösung verdünnten Plasmaprobe wurde schließlich $60 \mathrm{~min}$ mit $2 \mathrm{ng}\left[1,2-{ }^{3} \mathrm{H}_{2}\right]$ Dihydrotestosteron (spez. Akt. $1813 \mathrm{GBq} / \mathrm{mmol}$ ) bzw. $40 \mathrm{ng}\left[1,2-{ }^{3} \mathrm{H}_{2}\right]$ Cortisol (spez. Akt. $1702 \mathrm{GBq} / \mathrm{mmol}$ ) äquilibriert. Danach erfolgte am gleichen Tag die Agargelelektrophorese von $50 \mu$ dieser Lösung bei $4^{\circ} \mathrm{C}$. Bei einer Stromstärke von $110 \mathrm{~mA}$ ergab sich eine Spannung von etwa $360 \mathrm{~V}$; die Laufzeit bëtrug $90 \mathrm{~min}$ : Anschließend wurde die Agarplatte streifenweise miteinem Spezialgerät zerschnitten und die Radioaktivität (Tritium) nach Zugabe 
von $12 \mathrm{ml}$ Szintillationsflüssigkeit (Toluol-POPOP) in den einzelnen Agarstreifen gemessen. Die in den einzelnen Fraktionen nachgewiesene Radioaktivität wurde graphisch aufgetragen und auf diese Weise durch Flächenberechnung die an SHBG bzw. CBG gebundene Aktivität ermittelt. Unter Berücksichtigung der spezifischen Aktivität von tritiiertem Dihydrostestosteron bzw. Cortisol und der Serumverdünnung wurde daraus die Bindungskapazität des Serums für Dihydrotestosteron bzw. Cortisol nach den Angaben von Rosner (20) berechnet und in der Maßeinheit $\mu \mathrm{g} / \mathrm{l}$ oder $\mathrm{ng} / \mathrm{ml}$ angegeben. Die u.a. durch Temperaturschwankungen hervorgerufene Dissoziation der SHBG-Dihydrotestosteron-sowie CBG-Cortisol-Komplexe, die nach Wagner \& Rüffer Vortrag 20. Symp. Dtsch. Ges. Endokrinol. 1974) keinen größeren interindividuellen Unterschieden unterliegt, wurde bei der Berechnung der Bindungskapazitäten nicht berücksichtigt.

Mehrfachbestimmungen der SHBG-Bindungskapazität an verschiedenen Tagen ergaben einen Variationskoeffizienten von $11 \%$ im unteren Normalbereich $(\bar{x}=12,0 \mu \mathrm{g} / 1 ; n=11)$ und von $7 \%$ im pathologisch erhöhten Meßbereich $(\bar{x}=24,4 \mu \mathrm{g} / 1$; $\mathrm{n}=10$ ).

\section{Ergebnisse}

\section{Normalbereiche}

Die Bindungskapazität von SHBG im Serum von 43 männlichen Normalpersonen (Alter 20-67 Jahre) betrug im Mittel 12,7 $\mu \mathrm{g} / 1$; die Standardabweichung 2,2 $\mu \mathrm{g} / 1$. Der daraus errechnete Normalbereich $(\bar{x} \pm 2 s)$ war $8,3-$ $17,1 \mu \mathrm{g} / 1$, der $\overline{\mathrm{x}} \pm 3 \mathrm{~s}$-Bereich lag $\mathrm{zwischen} 6,1$ und $19,3 \mu \mathrm{g} / 1$. Bei den Normalpersonen im Alter über 40 Jahre waren die Mittelwerte etwas höher als bei den jüngeren Versuchspersonen (14,5 gegenüber $12,1 \mu \mathrm{g} / \mathrm{l})$. Die mittlere Bindungskapazität von CBG im Serum von 42 Normalpersonen betrug $64,6 \mu \mathrm{g} / 1$. Der Normalbereich lag zwischen 46,4-82,8 $\mu \mathrm{g} / 1$, der $\overline{\mathrm{X}} \pm 3 \mathrm{~s}$-Bereich war 37,3-91,9 $\mu \mathrm{g} / 1$. Eine Altersabhängigkeit war nicht erkennbar.

Werte außerhalb des $\pm 3 \mathrm{~s}$-Bereichs wurden als eindeutig pathologisch angesehen. Werte, die außerhalb des $\pm 2 \mathrm{~s}$ Bereichs, aber innerhalb des $\pm 3 \mathrm{~s}$-Bereichs lagen, galten als "suspekt hoch" bzw. "suspekt niedrig".

\section{SHBG bei Lebercirrhose}

Die SHBG-Bindungskapazität wurde bei 32 Patienten der Medizinischen Universitätsklinik und bei 31 Patienten der Chirurgischen Universitätsklinik Bonn bestimmt. Bei diesen 63 Männern mit Lebercirrhose war die Bindungskapazität von SHBG statistisch signifikant höher ( $\mathrm{p}<$ 0,001 im t-Test nach Student) als bei den 43 Normalpersonen $(\bar{x}=18,1$ und $s= \pm 5,4$ gegenüber $\bar{x}=12,7$ und $s= \pm 2,2 \mu \mathrm{g} / 1)$. Im einzelnen war dị SHBG-Bindungskapazität bei jeweils 14 Patienten aus beiden Kollektiven erhöht (außerhalb des \pm 3 s-Bereiches), bei 3 bzw. 4 Patienten lag SHBG im suspekten Bereich zwischen der $\pm 2 s$ and \pm 3 s-Grenze. Bei den übrigen 28 Patienten mit Lebercirrhose bewegte sich die SHBG-Bindungskapazität im Normalbereich $(-2 s$ bị $+2 s)$.

Bei 22 Patienten wurde Alkoholabusus und bei 14 Patienten eine vorausgegangene Hepatitis als Ursache der Lebercirrhose angesehen; in den übrigen Fällen waren ent- weder Alkoholabusus und eine Hepatitis aus der Anamnese bekannt, oder es war keine Zuordnung zu ätiologischen Faktoren möglich. Bei diesen Patienten ergab sich kein Anhalt dafür, daß bei Lebercirrhose nach Alkoholabusus endokrine Symptome wie Gynäkomastie und Hodenatrophie häufiger auftreten als bei der posthepatitischen Lebercirrhose. Wenn auch in der Hepatitis-Gruppe ein erhöhtes SHBG häufiger nachgewiesen werden konnte (in 7 von 14 Fällen) als in der Alkohol-Gruppe ( 7 von 22 Fällen, sowie 2 Patienten im suspekten Bereich), so waren die Unterschiede in beiden Gruppen jedoch nicht signifikant. Auch ergab sich kein Anhalt für eine Abhängigkeit der SHBG-Bindungskapazität vom Alter der Patienten oder von der Dauer der Erkrankung. Ob die Erkrankung kompensiert oder dekompensiert war, spielte für die Höhe der SHBG-Bindungskapazität keine Rolle. Bei Patienten, bei denen eine portocavale oder splenorenale Anastomose angelegt worden war, wurden sowohl normale als auch erhöhte Werte für SHBG festgestellt.

Korrelationen zwischen den Bindungskapazitäten von SHBG auf der einen Seite und der Gesamt-Eiweißkonzentration sowie den Aktivitäten der Cholinesterase und der Gerinnungsfaktoren V und VII im Serum als weiteren Kenngrößen der Proteinsynthese in der Leber auf der anderen Seite waren nicht erkennbar.

Eine beidseitige Gynäkomastie verschiedener Ausprägung wurde bei 27 Patienten festgestellt. Sie war bei den Patienten mit erhöhtem SHBG relativ häufiger als bei solchen mit normalem SHBG zu beobachten; von 26 Patienten mit erhöhtem SHBG wiesen 18 eine Gynäkomastie auf, während bei 24 Patienten mit normalem SHBG nur in 8 Fällen eine Gynäkomastie festgestellt wurde. Von 8 Patienten mit suspekten SHBG-Werten litt nur einer an Gynäkomastie. Bei den 27 Patienten mit Gynäkomastie war die SHBG-Bindungskapazität im Mittel nur geringfügig (nicht signifikant) höher ( $\bar{x}=19,5 \mu \mathrm{g} / \mathrm{l} ; \mathrm{s}= \pm 6,3$ ) als bei 29 Patienten mit Lebercirrhose ohne Gynäkomastie $(\overline{\mathrm{x}}=17,0 \mu \mathrm{g} / \mathrm{l} ; \mathrm{s}= \pm 5,3)$.

Bei 27 von 32 Patienten aus der Medizinischen Klinik wurde das Hodenvolumen, wie anfangs angegeben, berechnet. 15 Patienten hatten kleine Hoden $(<15 \mathrm{ml}$ Volumen; im folgenden als Hodenatrophie bezeichnet). Die Diagnose einer Hodenatrophie wurde in ausgewählten Fällen nach Aufklärung und mit Einverständnis der Patienten histologisch bestätigt. Ein Zusammenhang zwischen einer erhöhten SHBG-Bindungskapazität und dem Vorkommen einer Hodenatrophie war nicht sicher erkennbar. Die SHBG-Mittelwerte der Patienten mit bzw. ohne Hodenatrophie waren statistisch nicht signifikant unterschiedlich $(18,1$ gegenüber $17,6 \mu \mathrm{g} / \mathrm{l})$. Darüber hinaus war das mittlere Hodenvolumen - unabhängig vom Vorhandensein oder Fehlen einer Gynäkomastie jeweil's bei 12 Patienten gleich: 14,2 gegenüber $15,0 \mathrm{ml}$.

Potenz- und/oder Libidostörungen wurden in den meisten Fällen ( 25 von 30 befragten Patienten) ange- 
geben, bei Lebercirrhose-Kranken mit erhöhter SHBGBindungskapazität sogar in 12 von 13 Fällen.

Bei den Symptomen Spider naevi und Palmarerythem konnte keine Beziehung zwischen der SHBG-Bindungskapazität und dem qualitativen und quantitativen Auftreten dieser Symptome festgestellt werden.

Behaarungsanomalien (Verminderung der Achsel- und Stammbehaarung sowie des Bartwuchses, Veränderung der Schambehaarung im Sinne des weiblichen Typs) verschiedener Ausprägung wurden oft beobachtet bzw. anamnestisch angegeben. Sie waren insgesamt bei Patienten mit erhöhtem SHBG häufiger.

Spätere Kontrolluntersuchungen anläßlich ambulanter Nachuntersuchungen oder erneuter stationärer Aufnahmen ergaben durchweg einen Anstieg der Bindungskapazität des SHBG. Unter der Annahme einer kontinuierlichen Veränderung von SHBG errechnet sich ein mittlerer Anstieg um knapp ein Drittel (31\%) des Ausgangswertes innerhalb von 2 Monaten (Abb. 1). Während in einigen Fällen (Nr. 1-4) eine Besserung der Leberfunktion innerhalb dieses Zeitraumes registriert worden war, konnte in den übrigen Fällen retrospektiv hierzu nicht mehr sicher Stellung genommen werden.

Fast alle Patienten wurden zur Zeit der Untersuchung oder in den vorausgegangenen Wochen mit Vitaminund Verdauungsenzym-Präparaten sowie mit Spironolacton (meist $200 \mathrm{mg}$ Aldactone täglich) behandelt. $\mathrm{Da}$ im Gegensatz zu den erstgenannten Präparaten im Falle des Spironolactons ein Einfluß auf die hepatische Synthese von SHBG nicht ausgeschlossen werden

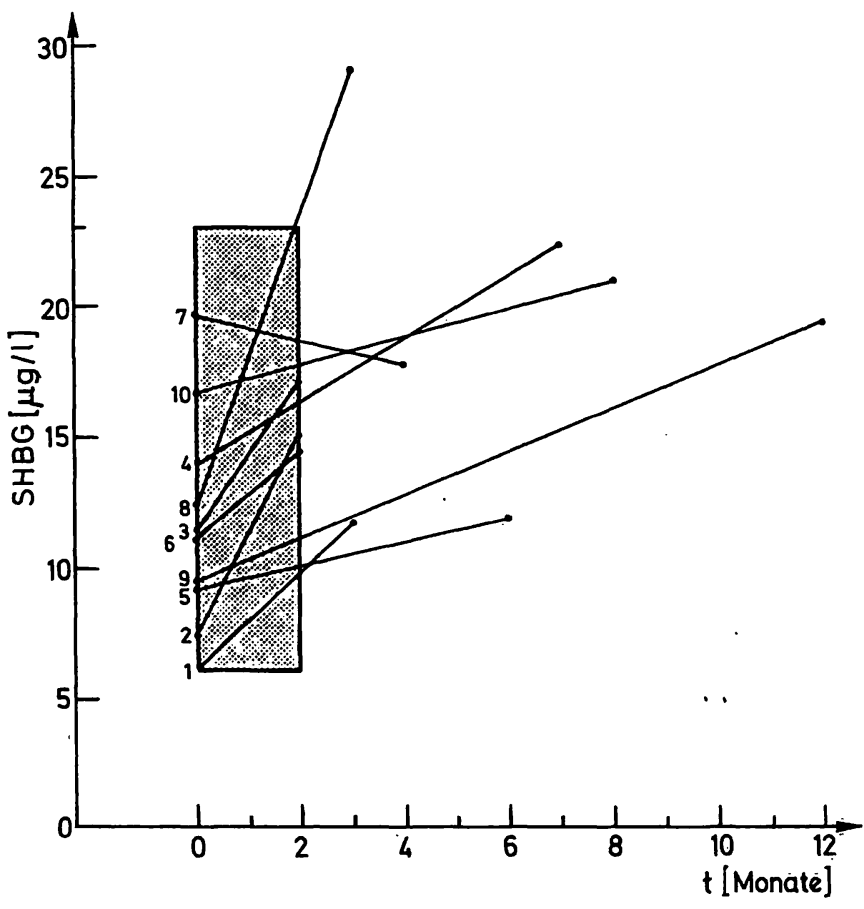

Abb. 1. Verhalten der Bindungskapazität von SHBG bei 10 Patienten mit Lebercirrhose in Abhängigkeit von der Zeit. Der Zeitraum 0 bis 2 Monate ist besonders gekennzeichnet.
Tạb. 1. Verhạtten der Bindungskapazität von SHBG und CBG vor (I) und nach (II) einer 7 tägigen Behandlung mit täglich $200 \mathrm{mg}$ Spironolacton.

\begin{tabular}{|c|c|c|c|c|c|}
\hline \multirow{2}{*}{$\begin{array}{l}\text { Versuchs- } \\
\text { person }\end{array}$} & \multirow{2}{*}{$\begin{array}{l}\text { Alter } \\
\text { (Jahre) }\end{array}$} & \multicolumn{2}{|c|}{ SHBG $[\mu \mathrm{g} / 1]$} & \multicolumn{2}{|c|}{ CBG. [ $\mu \mathrm{g} / \mathrm{l}]$} \\
\hline & & I & II & & II \\
\hline E1. & 20 & 14,8 & 16,3 & 75,5 & 76,6 \\
\hline Ko. & 24 & 10,6 & 12,6 & 69,3 & 59,5 \\
\hline Dö. & 25 & 12,4 & 12,4 & 64,2 & 66,6 \\
\hline Du. & 25 & 17,5 & 18,8 & 71,0 & 70,5 \\
\hline $\mathrm{Fa}$. & 25 & 14,2 & 13,2 & 60,7 & 68,4 \\
\hline Lo. & 25 & 12,8 & 11,3 & 74,6 & 70,5 \\
\hline Sä. & 26 & 12,5 & 12,9 & 62,9 & 70,9 \\
\hline $\mathrm{Fe}$ & 32 & 11,8 & 13,3 & 58,4 & 65,4 \\
\hline Su. & 33 & 16,0 & 15,6 & 70,5 & 69,4 \\
\hline$\overline{\mathbf{x}}$ & & 13,6 & 14,0 & 67,5 & 68,6 \\
\hline $\mathbf{s}$ & & $\pm 2,2$ & $\pm 2,4$ & $\pm 6,1$ & $\pm 4,7$ \\
\hline
\end{tabular}

kann, untersuchten wir bei neun männlichen, freiwilligen Versuchspersonen (20-33 Jahre alt) die Bindungskapazität von SHBG und $C B G$ vor und nach einer Woche Behandlung mit täglich $200 \mathrm{mg}$ Aldactone. Die gemessenen Werte sind in der Tabelle 1 dargestellt; signifikante Veränderungen waren nicht feststellbạ.

\section{CBG bei Lebercirrhose}

Bei insgesamt 63 Patienten mit Lebercirrhose wurde die CBG-Bindungskapazität bestimmt; 32 Patienten wurden in der Medizinischen und 31 Patienten in der Chirurgischen Univ.-Klinik Bonn behandelt. Die CBG-Bindungskapazität war bei den Patienten mit Lebercirrhose $(\overline{\mathrm{x}}=$ $49,7 ; s= \pm 15,9)$ statistisch signifikant niedriger $(p<$ $0,001)$ als bei den gesunden Versuchspersonen $(\bar{x}=64,6$; $s= \pm 9,1)$. Etwas mehr als die Hälfte der Patienten hatte jedoch eine CBG-Bindungskapazität im Normalbereich. Bei jeweils 15 Patienten lag CBG im suspekten bzw. pathologischen Bereich unterhalb der -2s-Grenze. Eine genaue Analyse der Werte ergab, daß von 28 Patienten mit erhöhter SHBG-Bindungskapazität nur vier eine erniedrigte CBG-Bindungskapazität aufwiesen, währènd bei ebenfalls 28 Patienten mit normalem SHBG in 11 Fällen ein erniedrigtes CBG gefunden wurde. Umgekehrt war bei 15 Patienten mit erniedrigter CBG-Bindungskapazität in 11 Fällen SHBG normal, und bei 33 Patienten mit normalem CBG war SHBG in 17 Fällen erhöht.

Der Versuch, 69 Wertepaare der Bindungskapazitäten von CBG und SHBG bei 63 Patienten miteinander zu korrelieren, ergab keine statistisch signifikante Korrelation in der Plasmakonzentration dieser beiden Transportproteine. Von besonderem Interesse erscheinen jedoch die Fälle mit normalem SHBG bei gleichzeitig erniedrigtem CBG. Im t-Test nach Student ergab sich, daß Lebercirrhoșe-Kranke mit erniedrigter CBG-Bindungskapażität - unäbhängig vom Alter, vón dèr Dauer und vom Schweregrad der klinischen Symptome - eine statistisch-signifikant niedrigere SHBG-Bindungskapazität aufwiesen $(p<0,005)$ als die Patienten mit einer CBG-Bindungskapażität im Normaḷbereich. Darüber 
hinaus wurde geprüft, ob Zusammenhänge zwischen CBG einerseits und den Aktivitäten der Cholinesterase und dem Gerinnungsfaktor VII als weiteren Kenngrößen der Proteinsynthese in der Leber bestehen. Es zeigte sich, daß auch zwischen diesen Meßgrößen keine signifikante Korrelation ( $r=0,44$ bzw. $r=0,51$ ) nachweisbar war.

Aus diesen Befunden ergibt sich kein Anhalt dafür, daß die Ätiologie der Lebercirrhose von Bedeutung für die Bindungskapazität von $C B G$ sein könnte; erniedrigte CBG-Werte wurden gleich häufig bei der posthepatischen wie bei der Alkoholcirrhose gefunden. Auch der Schweregrad der Erkrankung (kompensiert vs. dekompensiert) scheint keinen Einfluß auf die Höhe der Bindungskapazität von CBG zu haben.

Kausale Beziehungen zwischen der Höhe der Bindungskapazität des CBG und den verschiedenen endokrinen Symptomen bei Lebercirrhose waren nicht zu erwarten; es zeigte sich allerdings, daß Patienten mit niedrigem CBG weniger häufig eine Gynäkomastie aufwiesen als Patienten mit normalem CBG. Die Unterschiede in der Bindungskapazität von CBG bei Patienten mit bzw. ohne Gynäkomastie waren jedoch statistisch nịcht signifikant ( 51,7 gegenüber $47,3 \mu \mathrm{g} / \mathrm{l} ; \mathrm{s}= \pm 15,6$ bzw. 15,$9 ; \mathrm{n}=27$ bzw. 26).

Bei neun Patienten wurde anläßlich von Nachuntersuchungen zwei bis zwölf Monate später erneut Blut zur

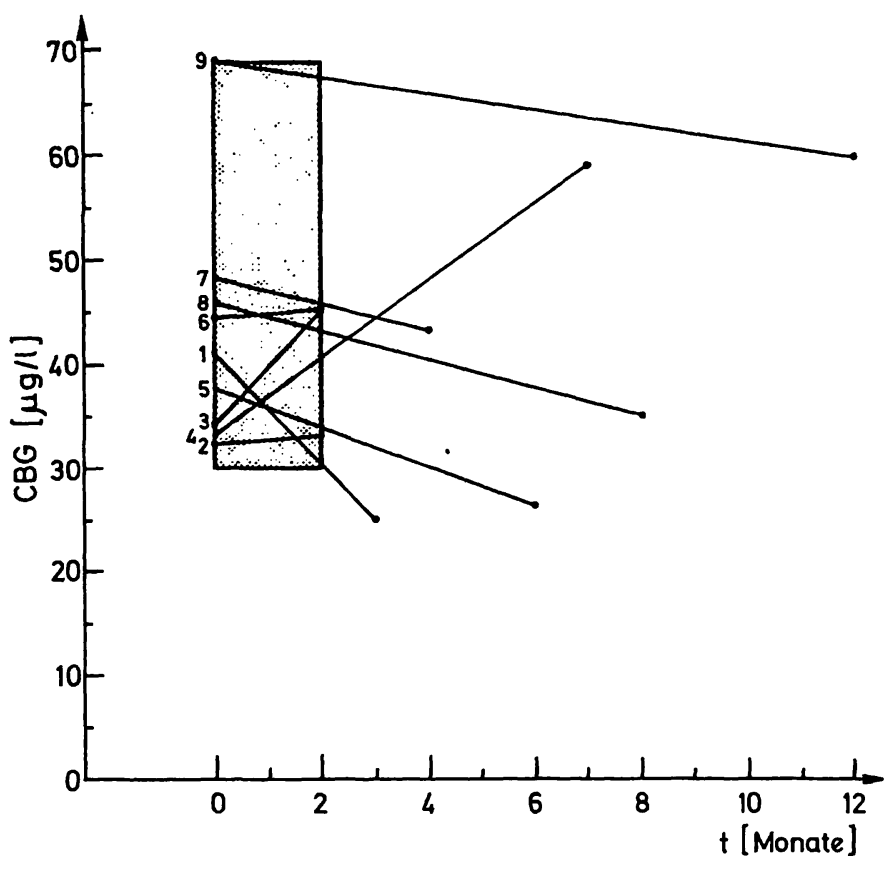

Abb. 2. Verhalten der Bindungskapazität von $C B G$ bei neun Patienten mit Lebercirrhose in Abhängigkeit von der Zeit. Der Zeitraum 0 bis 2 Monate ist besonders gekennzeichnet.

Bestimmung von SHBG (vgl. Abb. 1) und CBG (Abb. 2) entnommen. Die Bindungskapazität von $C B G$ war jedoch nicht in charakteristischer Weise verändert.
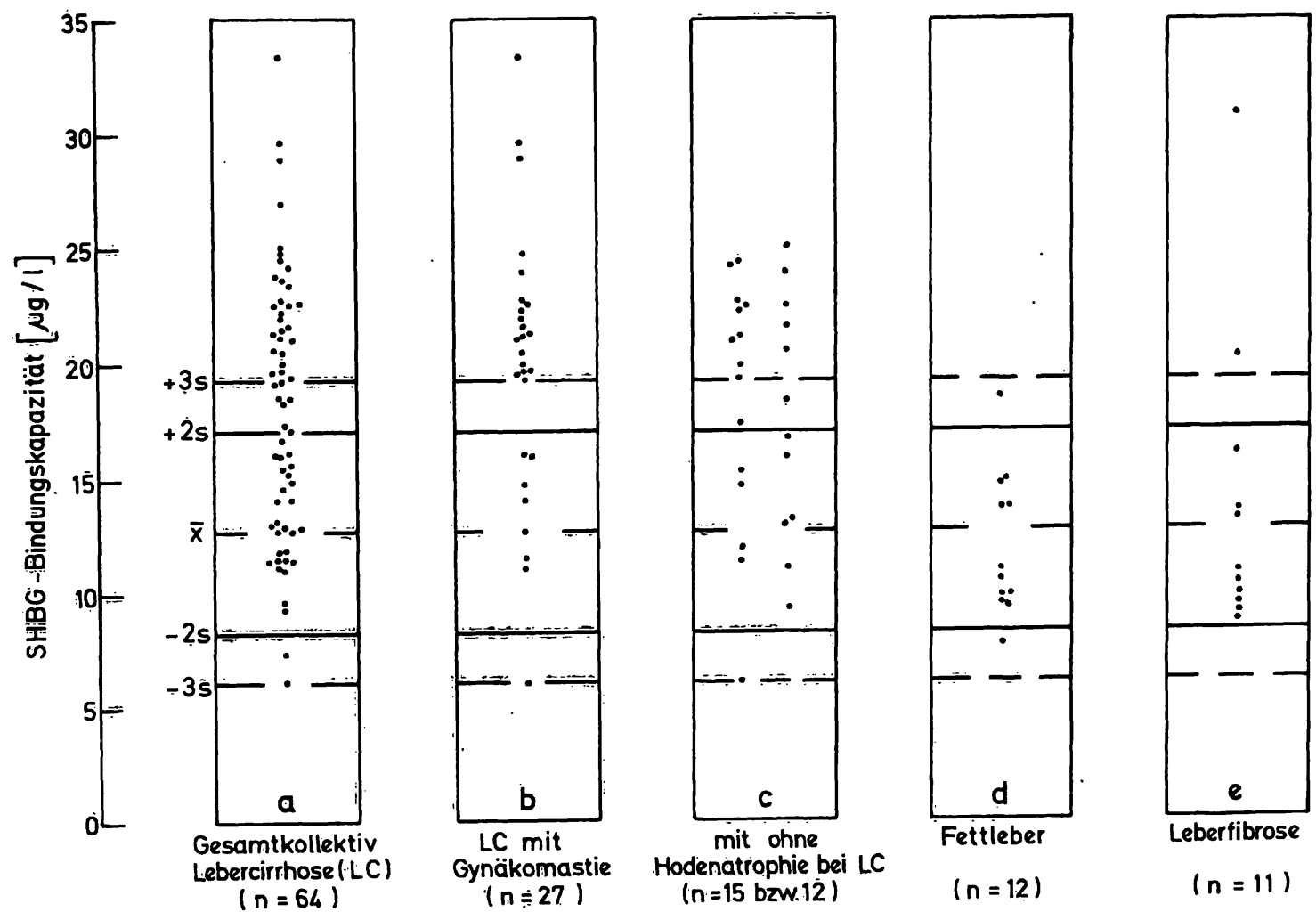

Abb 3. Punktediagramm der Bindungskapazität von SHBG bei a) 64 Patienten mit Lebercirrhose, b) 27 Patienten mit Gynäkomastie aus diesem Kollektiv, c) Patienten mit und ohne Hodenatrophie bei Lebercirrhose, d) 12 Patienten mit Fettleber und e) 11 Patienten mit Fibrose der Leber.

Die waagerechten Linien zeigen den arithmetischen Mittelwert $\overline{\mathbf{x}}$ sowie die doppelte und dreifache Standardabweichung der SHBG-Bindungskapazität bei den Normalpersonen an. 
Bindungskapazität von SHBG und CBG bei Fettleber und toxischer Fibrose der Leber

Bei 12 Patienten mit Fettleber (Alter 31-56 Jahre; in 9 Fällen durch Alkoholabusus) und bei 11 Patienten mit Fibrose der Leber (bei Polyvinylchlorid-Krankheit; Alter 31-56 Jahre) wurden ebenfalls SHBG (Abb. 3) und CBG (Abb. 4) bestimmt. Der t-Test nach Student ergab bei diesen beiden Leberkrankheiten keine statistisch signifikanten Unterschiede in der Bindungskapazität dieser Transportproteine gegenüber den gesunden Vergleichspersonen.

Während bei Fettleber in keinem Fall eindeutige Veränderungen in der Bindungskapazität von SHBG oder CBG nachgewiesen werden konnten, zeigten jeweils zwei (verschiedene) Patienten mit Fibrose der Leber ein erhöhtes SHBG bzw. ein erniedrigtes CBG.

\section{Diskussion}

Die vorliegenden Ergebnisse bestätigen und erweitern die schon von anderen Patienten und Autoren beschriebene Beobachtung, wonach die Bindungskapazität von SHBG bei Patienten mit Lebercirrhose erhöht ist (13, 17, 19-21). Allerdings stellte sich bei der genaueren

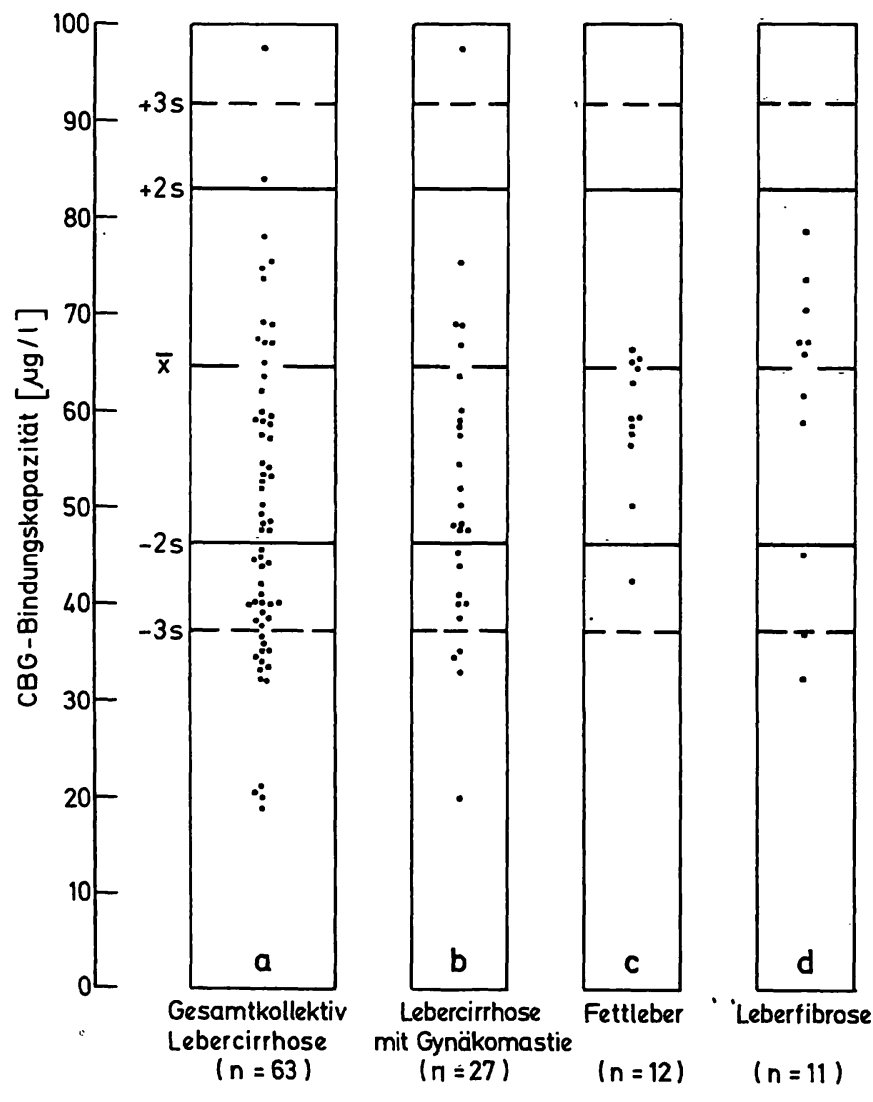

Abb. 4. Punktediagramm der Bindungskapazität von $\mathrm{CBG}$ bei a) 63 Patienten mit Lebercirrhose, b) 27 Patienten mit Gynäkomastie aus diesem Kollektiv, c) 12 Patienten mit Fettleber und d) 11 Patienten mit Leberfibrose. Die waagerechten Linien zeigen den arithmetischen Mittelwert $\overline{\mathbf{x}}$ sowie die doppelte und dreifache Standardabweichung der CBG-Bindungskapazität bei den Normalpersonen an.
Untersuchung heraus, daß diese Erhöhung nur bei etwa der Hälfte der männlichen Patienten nachweisbar war: nur 28 von 63 Patienten hatten eine eindeutig erhöhte und weitere sieben eine suspekt hohe SHBG-Bindungskapazität. Diese scheint unabhängig von der Ätiologie der Lebercirrhose, vom Alter der Patienten und von der Dauer der Krankheit zu sein. Von anderer Seite (25) war die Meinung vertreten worden, daß Alkohol selbšt zu Störungen im Androgenstoff wechsel (Hemmung der Testosteronbiosynthese und auch der Spermatogenese) führt, so daß bei alkoholbedingter Lebercirrhose häufiger endokrine Ausfallserscheinungen zu erwarten wären. Diese Vermutung ließ sich an unserem Kollektiv nicht bestätigen. Korrelationen zu Leberfunktionsstörungen, insbesondere zu Parametern der Proteinsynthese in der Leber, konnten nicht aufgezeigt werden.

Bisher wụrde unseres Wissens nur in einer größeren Studie (8) versucht, Beziehungen zwischen den pathobiochemischen Veränderungen und den klinisch manifesten Androgenmangelsymptomen nachzuweisen; dabei stellte sich herauss, daß zwischen den Veränderungen in der Bindungskapazität des SHBG und den gleichzeitig beschriebenen niedrigen Testosteron- und hohen Östradiol-Konzentrationen im Plasma keine signifikanten Korrelationen bestanden. Wie auch in unserer Untersuchung konnte keine Abhängigkeit der hormonalen Veränderungen und der endokrinen Symptome vom Schweregrad der Leberkrankheit nachgewiesen werden.

Bei neun von 10 Lebercirrhose-Kranken war im Zeitraum von 2-12 Monaten nach der Erstuntersuchung der Anstieg der Bindungskapazität von SHBG zunächst als mögliche Folge der Behandlung mit Spironolacton gedeutet worden. Deshalb wurde versucht, diese Hypothese durch ein Zusatzexperiment zu bestätigen oder zu verwerfen; dabei zeigte sich, daß zumindest eine kurzfristige Behandlung gesunder, junger Probanden mit Spironolacton in mittlerer Dosierung keine Veränderungen in den Bindungskapazitäten von SHBG oder CBG erzeugt (Tab. 1).

Im Gegensatz zu SHBG ist die Bindungskapazität von CBG bei Lebercirrhose unseres Wissens bisher nur von einer Arbeitsgruppe bei 15 männlichen Patienten untersucht worden (22); mit der Methode der Gleichgewichtsdialyse wurden von diesen Autoren keine signifikanten Änderungen der Bindungskapazität von CBG festgestelit. Anhand unseres Kollektivs von 63 Männern mit Lebercirrhose konnte hingegen eine statistisch signifikante Erniedrigung $(p<0,001)$ nachgewiesen werden, obgleich eindeutig erniedrigte oder suspekt niedrige Werte nur bei jeweils 15 Patienten bestimmt wurden. Von Interesse ist die Beobachtung, daß die Bindungskapazität von SHBG bei Patienten mit niedrigem CBG geringer war als bei solchen mit normalem CBG. Korrelationen zwischen der Höhe von CBG und der Ausprägung der Androgenmangelsymptome konnten weder von Sandberg \& Slaunwhite (22) noch von uns aufgezeigt wérden, so 
daß die klinische Relevanz dieser Befunde ungeklärt bleibt. Kley et al. (10) hatten bei Lebercirrhose (15 Männer) weder Veränderungen in der Gesamtkonzentration noch in der prozentualen Bindung von Cortisol im Plasma nachweisen können.

Wie schon Kley et al. (10) beobachtet hatten, scheint die prozentuale Bindung der Sexualhormone und von Cortisol bei Patienten mit einer Fettleber nicht verändert zu sein. Auch bei Vorliegen einer Fibrose der Leber (hier bei PVC-Krankheit) konnten wir keine charakteristischen Veränderungen der Bindungskapazitäten von SHBG und CBG nachweisen. Da bei neun der 12 Patienten mit Fettleber ein chronischer Alkoholabusus bekannt war, könnte diese Beobachtung ebenfalls dafür sprechen, daß Alkohol per se zu keinem Anstieg der SHBG-Bindungskapazität führt.

Es bestehen keine Zweifel, daß eine erhöhte Bindungskapazität von SHBG im Serum bei Lebercirrhose das Gleichgewicht zwischen Testosteron/Dihydrotestosteron und Östradiol-17 $\beta$ zu Ungunsten der Androgene verschieben kann. Die fehlende Korrelation zwischen SHBG und der Schwere der Erkrankung sowie der Ausprägung der endokrinen Symptome spricht jedoch dafür, daß weitere Störungen des Sexualendokriniums vorliegen müssen. So ist bekannt, daß sowohl die Umwandlung von Testosteron zu 4-Androstendion $(26,27)$ als auch diejenige von 4-Androstendion zu Östron $(27,28)$ bei Patienten mit Lebercirrhose deutlich erhöht ist. Die Auswirkungen der vermehrten peripheren Konversion von Androgenen zu Östrogenen können durch eine gleichzeitig erhöhte SHBG-Bindungskapazität verstärkt werden; offenbar reichen die durch eine erhöhte Synthese von SHBG bedingten Verschiebungen zwischen Androgenen und Östrogenen allein nicht aus, um Symptome wie Gynäkomastie und Hodenatrophie zu erzeugen. Dies würde erklären, warum manche Patienten trotz deutlich erhöhter SHBG-Bindungskapazität keine Zeichen des Hypogonadismus aufweisen, andere mit normalem SHBG solche Symptome aber zeigen. Die Frage, inwieweit die erhöhte Produktion von Östron und Prolaktin (29) die Ausprägung der Gynäkomastie zusätzlich beeinflußt, kann zur Zeit noch nicht beantwortet werden.

\section{Danksagung}

Herrn Professor Dr. P. W. Jungblut und Herrn Dr. R. K. Wagner, Max-Planck-Institut für Zellbiologie, Wilhelmshaven, danken wir für die Einarbeitung in die Methodik der Agargelelektrophorese.

\section{Literatur}

1. Breuer, H., Rohner, H. G., Lafosse, M.\& Kaulhausen, H. (1975), diese Z. 13, 250.

2. Edmondson, H. A., Glass, S. J. \& Soll, S. N. (1939), Proc. Soc. Exp. Biol. Med. 42, 97-99.

3. Glass, S., Edmondson, H. A. \& Soll, S. N. (1940), Endocrinology 27, 749-752.

4. Cameron, C. B. (1957), J. Endocrinol. 15, 199-205.

5. Lloyd, C. W. \& Williams, R. H. (1948), Amer. J. Med. 4, 315-330.

6. Rupp, J., Cantarow, A., Rakoff, A. E. \& Paschkis, K. E. (1951), J. Clin. Endocrinol. Metab. 11, 688-699.

7. Zondek, B. \& Black, R. (1947), J. Clin. Endocrinol. Metab. 7, 519-529.

8. Baker, H. W. G., Burger, H. G., de Kretser, D. M., Dulmanis, A., Hudson, B., O'Connor, S., Paulsen, C. A., Purcell, N., Rennie, G. C., Seah, C. S., Taft, H. P. \& Wang, C. (1976), Quarterly J. Med. 45, 145-178.

9. Chopra, I. J., Tulchinsky, D. \& Greenway, F. L. (1973), Ann. Intern. Med. 79, 198-203.

10. Kley, H. K., Nieschlag, E., Wiegelmann, W., Solbach, H. G. \& Krüskemper, H. L. (1975), Acta Endocrinol. (Kbh.) $275-285$.

11. Korenman, S. G., Perrin, L. E. \& McCallum, T. (1969), J. Clin. Invest. 48,45 a.

12. Vermeulen, A., Mussche, M. \& Verdonck, L. (1972), Ex= cerpta Med. Int. Congr. Ser. 256, 305 (abstract).

13. Galvao-Teles, A., Anderson, D. C., Burke, C. W., Marshall, J. C., Corker, C. S., Brown, R. L. \& Clark, M. L. (1973), Lancet $I, 173-177$.

14. Coppage, W. S. \& Cooner, A. E. (1965), New Engl. J. Med. 273, 902-907.
15. Kent, J. R., Scaramuzzi, R. J., Lauwers, W., Parlow, A. F., Hill, M., Penardi, R. \& Hilliard, J. (1973), Gastroenterology 64, 111-115.

16. Vermeulen, A., Rubens, R. \& Verdonck, L. (1972), J. Clin. Endocrinol. Metab. 34, 730-735.

17. Tavernetti, R. R., Rosenbaum, W., Kelly, W. G., Christy, N. P. \& Roginsky, M. S. (1967), J. Clin. Endocrinol. Metab. $27,920-926$.

18. Burke, C. W. \& Anderson, D. C. (1972), J. Endocrinol. 53, XXVI-XXVII.

19. Breuer, J., Schneider, H. Th. \& Breuer, H. (1970), diese Z. 8, $626-631$.

20. Rosner, W. (1972), J. Clin. Endocrinol. Metab. 34, 983-988.

21. Vermeulen, A., Verdonck, L., van der Straaten, M. \& Orie, N. (1969), J. Clin. Endocrinol. Metab. 29, 1470-1480.

22. Sandberg, A. A. \& Slaunwhite, W. R. (1959), J. Clin. Invest. $38,1290-1297$.

23. Prader, A. (1966), Triangel 7, 240.

24. Wagner, R. K. (1972), Hoppe-Seyler's Z. Physiol. Chem. 353, $1235-1245$.

25. Van Thiel, D. H. \& Lester, R. (1974), New Engl. J. Med. 291, 251-253.

26. Gordon, G. G., Olivo, J., Rafii, F. \& Southren, A. L. (1975), J. Clin. Endocrinol. Metab. 40, 1018-1026.

27. Thijssen, J. H. H., Lourens, J. \& Donker, G. H. (1974), Acta Endocrinol. (Kbh.) Suppl. 184, 31.

28. MacDonald, P. C., Grodin, J. M. \& Siiteri, P. K. (1971) in Control of gonadal steroid secretion (Baird, D. T. \& Strong, J. A., eds.), University Press, Edinburgh, p. 158.

29. Van Thiel, D. H., Gavaler, J. S. \& Lester, R. (1975), Gastroenterology 68, A-77/934.

Prof. Dr. H. Breuer

Institut für Klinische Biochemie der Universität Venusberg

D-5300 Bonn 1 
